\title{
Spin dynamics in highly frustrated pyrochlore magnets
}

\author{
Sylvain Petit ${ }^{1, \text { a }}$, Solène Guitteny ${ }^{1}$, Julien Robert ${ }^{1}$, Pierre Bonville $^{2}$, Claudia Decorse $^{3}$, Jacques Ollivier $^{4}$, Hannu Mutka $^{4}$ \\ and Isabelle Mirebeau ${ }^{1}$ \\ ${ }^{1}$ Laboratoire Léon Brillouin, CEA Saclay, 91190 Gif-sur-Yvette, France \\ ${ }^{2}$ SPEC, CEA Saclay, 91190 Gif-sur-Yvette, France \\ ${ }^{3}$ ICMMO, Université Paris XI, 91405 Orsay, France \\ ${ }^{4}$ Institut Laue Langevin, 6 rue Jules Horowitz, BP. 156, 38042 Grenoble, France
}

\begin{abstract}
This paper aims at showing the complementarity between time-of-flight and triple-axis neutron scattering experiments, on the basis of two topical examples in the field of geometrical magnetic frustration. Rare earth pyrochlore magnets $\mathrm{R}_{2} \mathrm{Ti}_{2} \mathrm{O}_{7}$ ( $\mathrm{R}$ is a rare earth) play a prominent role in this field, as they form model systems showing a rich variety of ground states, depending on the balance between dipolar, exchange interactions and crystal field. We first review the case of the XY antiferromagnet $\mathrm{Er}_{2} \mathrm{Ti}_{2} \mathrm{O}_{7}$. Here a transition towards a Néel state is observed, possibly induced by an order-bydisorder mechanism. Effective exchange parameters can be extracted from $S(Q, \omega)$. We then examine the case of the spin liquid $\mathrm{Tb}_{2} \mathrm{Ti}_{2} \mathrm{O}_{7}$. Recent experiments reveal a complex ground state characterized by "pinch points" and supporting a low energy excitation. These studies demonstrate the existence of a coupling between crystal field transitions and a transverse acoustic phonon mode.
\end{abstract}

\section{Introduction}

Geometrically frustrated magnetism is a forefront research topic within condensed matter physics, as testified by the wealth of exotic phenomena discovered over the past years [1-3]. The inability of frustrated systems to satisfy all pairs of interactions simultaneously is reflected by the fact that an infinite number of different magnetic configurations minimize the classical energy, hence leading to an extensive degeneracy. This results in exotic spin dynamics, that can be studied by different means, such as magnetization, ac susceptibility, Mössbauer spectroscopy, muons and inelastic neutron scattering. For instance, quasi-elastic scattering, unraveling relaxation phenomena at short times scales as well as collective spin excitations from a given ground state (yielding the exchange interactions), or the continuum of fractionalized excitations, can be studied using state of art neutron instrumentation. Single crystal time-of-flight (TOF) data allow one to map out the reciprocal and energy space in great detail. As a result, it becomes possible to measure for instance the full dispersion of spin wave excitations, and, with the help of numerical simulations, to determine the relevant exchange constants. However, despite the high luminosity of such instruments, the counting time in one configuration (wavelength, magnetic field, temperature) is still of the order of about one day, so that measuring precisely the temperature, field dependence or the spin polarization analysis at a given $\mathrm{Q}$ position, remains the preserve of triple axis spectrometers (TAS). The suite of both spectrometers is thus clearly complementary.

a e-mail: sylvain.petit@cea.fr
It is the main objective of this paper to show these points, on the basis of recent experiments carried out on two pyrochlore magnets, namely $\mathrm{Er}_{2} \mathrm{Ti}_{2} \mathrm{O}_{7}$ and $\mathrm{Tb}_{2} \mathrm{Ti}_{2} \mathrm{O}_{7}$. In $\mathrm{Er}_{2} \mathrm{Ti}_{2} \mathrm{O}_{7}$, a transition towards a Néel state is observed, possibly induced by an order-bydisorder mechanism. $S(Q, \omega)$ can be confronted to theory and effective exchange parameters can be extracted. Next, we examine the case of the spin liquid material $\mathrm{Tb}_{2} \mathrm{Ti}_{2} \mathrm{O}_{7}$. Combination of TOF and polarized triple axis measurements demonstrate the existence of a low energy propagating excitation emanating from the spin liquid ground state. Anomalies of the phonon modes, as well as of the first crystal electric field (CEF) level, suggest a strong dynamical coupling to the lattice.

\section{2. $\operatorname{Er}_{2} \mathbf{T i}_{2} \mathbf{O}_{7}$}

Since a decade, the antiferromagnetic model for $\mathrm{XY}$ spins on the pyrochlore lattice has been considered with much interest since it displays an extensive classical degeneracy [4-6] along with classical and quantum order by disorder (ObD) effects [5-14]. ObD is a very important theoretical concept in frustrated magnetism, coming into play by selecting a particular configuration out of the degenerate manifold $[15,16]$. At finite temperature, the selection operates because fluctuations away from this configuration allow for a relative gain of entropy (classical ObD). At zero temperature, the selection can also operate because the contribution to the total energy of zero point fluctuations is minimum for this configuration (quantum ObD). Until now, the number of confirmed examples of such a mechanism in real materials has remained scarce

This is an Open Access article distributed under the terms of the Creative Commons Attribution License 4.0, which permits unrestricted use, distribution, and reproduction in any medium, provided the original work is properly cited. 

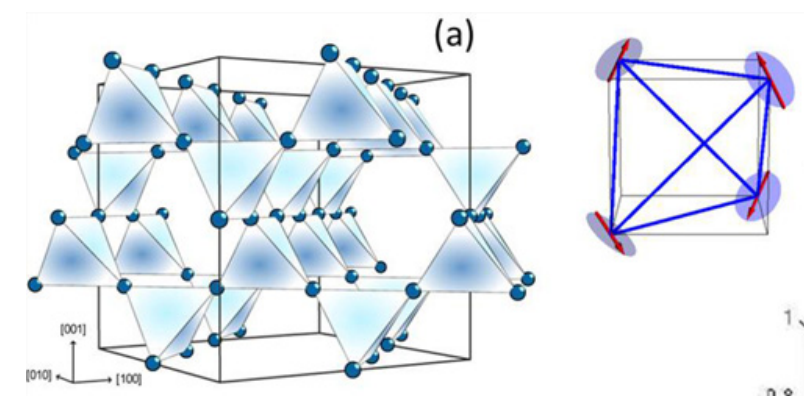

(b)
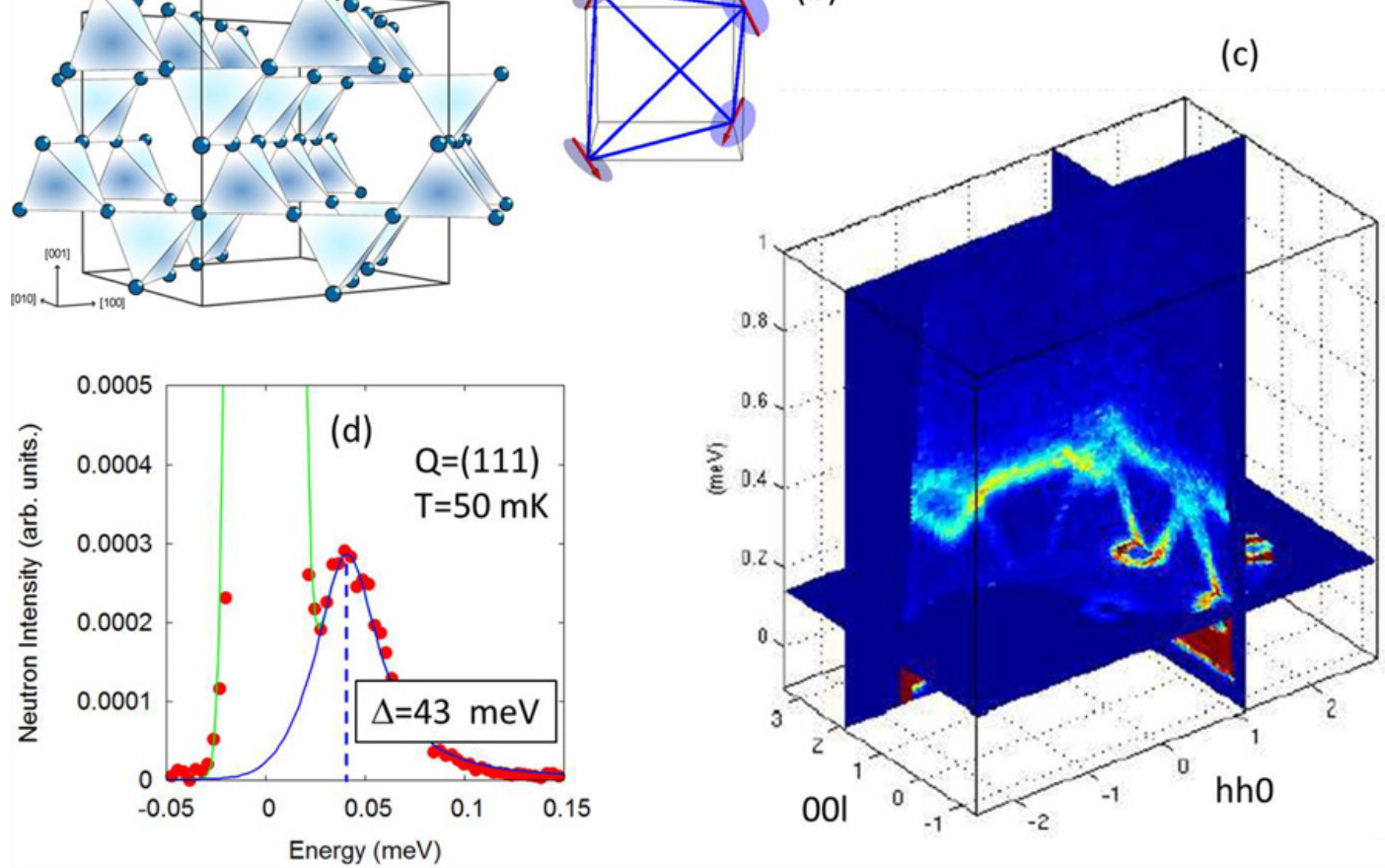

Figure 1. a) Sketch of the pyrochlore lattice. Rare earth ions only are shown for sake of clarity. b) Sketch of the $\psi_{2}$ configuration in $\mathrm{Er}_{2} \mathrm{Ti}_{2} \mathrm{O}_{7}$. c) INS data recorded at IN5, showing cuts in $(Q, \omega)$ space in the ordered phase of $\operatorname{Er}_{2} \mathrm{Ti}_{2} \mathrm{O}_{7}$. d) IN5 raw data recorded with an incident wavelength of $8.5 \AA$ at $Q=(111)$ and $T=50 \mathrm{mK}$ showing the spin gap (blue line) at $43 \mu \mathrm{eV}$.

[17]. However, $\mathrm{Er}_{2} \mathrm{Ti}_{2} \mathrm{O}_{7}$ has recently been proposed as a most convincing case of $\mathrm{ObD}[8-11]$.

In $\mathrm{Er}_{2} \mathrm{Ti}_{2} \mathrm{O}_{7}$, the CEF acting on the Kramers $\mathrm{Er}^{3+}$ ion is responsible for a strong XY-like anisotropy, with easy magnetic planes perpendicular to the local $\langle 111\rangle$ ternary axes $[2,6] . \mathrm{Er}_{2} \mathrm{Ti}_{2} \mathrm{O}_{7}$ undergoes a second order phase transition towards an antiferromagnetic noncollinear $\mathbf{k}=0$ Néel phase at $T_{\mathrm{N}}=1.2 \mathrm{~K}[14,18-20]$. The corresponding magnetic configuration, denoted $\psi_{2}$ is depicted in Fig. 1b [14,21].

The minimal model for this material takes into account an anisotropic bilinear exchange Hamiltonian written for the $\mathrm{Er}^{3+}$ moments $\vec{J}_{i}$ at sites $i$ of the pyrochlore lattice (see Refs. [22] and [23]) as well as the CEF contribution, $\mathcal{H}_{\mathrm{CEF}}$ :

$$
\mathcal{H}=\mathcal{H}_{\mathrm{CEF}}+\frac{1}{2} \sum_{i, j} \vec{J}_{i} \cdot \mathcal{K}_{i, j} \cdot \vec{J}_{j}
$$

where $\mathcal{H}_{\mathrm{CEF}}=\sum_{n, m} B_{n m} O_{n m}$ is written in terms of $O_{n m}$ Stevens operators [24-26]. $\mathcal{K}_{i, j}$ denotes an anisotropic exchange coupling tensor, defined in the $(\vec{a}, \vec{b}, \vec{c})$ frame attached to the $\mathrm{Er}^{3+}-\mathrm{Er}^{3+}$ bonds [27]. It consists in a symmetric part with 3 parameters $\mathcal{K}_{a, b, c}$, and an antisymmetric (Dzyaloshinskii-Moriya) part with constant $\mathcal{K}_{4}$.

It turns out that the ground CEF state is a doublet, very well separated from the first excited states. The above model can thus be projected onto the subspace spanned by the pseudospin $1 / 2$ that describes the two states of the ground doublet, yielding [9]:

$$
\begin{aligned}
\mathcal{H}= & \sum_{i, j} \mathrm{~J}_{z z} \mathrm{~S}_{i}^{z} \mathrm{~S}_{j}^{z}-\mathrm{J}_{ \pm}\left(\mathrm{S}_{i}^{+} \mathrm{S}_{j}^{-}+\mathrm{S}_{i}^{-} \mathrm{S}_{j}^{+}+\right) \\
& +\mathrm{J}_{ \pm \pm}\left(\gamma_{i j} \mathrm{~S}_{i}^{+} \mathrm{S}_{j}^{+}+\gamma_{i j}^{*} \mathrm{~S}_{i}^{-} \mathrm{S}_{j}^{-}\right) \\
& +\mathrm{J}_{z \pm}\left[\mathrm{S}_{i}^{z}\left(\zeta_{i j} \mathrm{~S}_{j}^{+}+\zeta_{i j}^{*} \mathrm{~S}_{j}^{-}\right)+i \leftrightarrow j\right] .
\end{aligned}
$$

In this model, the spins are written is their local bases $\left(\zeta_{i j}\right.$ and $\gamma_{i j}$ are geometrical coefficients, see [9]) and $\mathcal{K}_{i, j}$ is split in four anisotropic nearest-neighbor exchange parameters $\left(\mathrm{J}_{ \pm \pm}, \mathrm{J}_{ \pm}, \mathrm{J}_{z \pm}, \mathrm{J}_{z z}\right)$ with the following relations:

$$
\begin{aligned}
& \mathrm{J}_{z z}=\lambda_{z}^{2} \frac{\mathcal{K}_{a}-2 \mathcal{K}_{c}-4 \mathcal{K}_{4}}{3} \\
& \mathrm{~J}_{ \pm}=-\lambda_{\perp}^{2} \frac{2 \mathcal{K}_{a}-3 \mathcal{K}_{b}-\mathcal{K}_{c}+4 \mathcal{K}_{4}}{12} \\
& \mathrm{~J}_{z \pm}=\lambda_{\perp} \lambda_{z} \frac{\mathcal{K}_{a}+\mathcal{K}_{c}-\mathcal{K}_{4}}{3 \sqrt{2}} \\
& \mathrm{~J}_{ \pm \pm}=\lambda_{\perp}^{2} \frac{2 \mathcal{K}_{a}+3 \mathcal{K}_{b}-\mathcal{K}_{c}+4 \mathcal{K}_{4}}{12}
\end{aligned}
$$

where $\lambda_{\perp, z}=g_{\perp, z} / g_{J}, g_{\perp, z}$ being the Landé factors.

The set of exchange parameters has been determined by inelastic neutron scattering experiments carried out in a large applied magnetic field (see right column in Table 1). In zero field, theory predicts for these parameters a quantum $\mathrm{ObD}$ selection of the $\psi_{2}$ state [7-11]. The ObD mechanism also predicts the opening of a spin gap, yet smaller that experimental findings [29-31]. 
Table 1. Anisotropic exchange parameters in $\mathrm{Er}_{2} \mathrm{Ti}_{2} \mathrm{O}_{7}$. Units are in $10^{-2} \mathrm{meV}$. Positive values correspond to $\mathrm{AF}$ interactions.

\begin{tabular}{|c|c|c|}
\hline Coupling & Fit based on Hamiltonian (1) & Ref. [9] \\
\hline $\mathrm{J}_{ \pm \pm}$ & $4.54( \pm 0.1)$ & $4.2( \pm 0.5)$ \\
$\mathrm{J}_{ \pm}$ & $5.84( \pm 0.1)$ & $6.5( \pm 0.75)$ \\
$\mathrm{J}_{z \pm}$ & $0.92( \pm 0.1)$ & $-0.88( \pm 1.5)$ \\
$\mathrm{J}_{z z}$ & $-0.87( \pm 0.1)$ & $-2.5( \pm 1.8)$ \\
\hline
\end{tabular}

In a recent work, the impact of the projection onto the spin $1 / 2$ subspace was examined to see if $\mathrm{ObD}$ is also at play when using the full CEF Hamiltonian of Eq. (1). The spin excitation spectra was calculated within the Random Phase approximation [26,32,33]. New neutron measurements were performed on a large $\mathrm{Er}_{2} \mathrm{Ti}_{2} \mathrm{O}_{7}$ single crystal grown with the floating zone technique. The crystal was inserted in a copper sample holder and attached on the cold finger of a dilution fridge, allowing the sample to be cooled down to $50 \mathrm{mK}$. Data were collected on the IN5 time-of-flight instrument (ILL) in its single crystal set-up, with an incident neutron wavelength of $6 \AA$. Spin excitation spectra measured in $(Q, \omega)$ space at $50 \mathrm{mK}$ are shown in Fig. 1. These new data allow one to determine a new set of anisotropic exchange parameters, see left column in Table 1 . These values are more precise than those determined in Ref. [9], but are quite compatible with them, except $\mathrm{J}_{z \pm}$ which is somewhat different. The present data also confirm the opening of a spin gap at zone centers: $\Delta_{G}^{\exp } \approx 43 \mu \mathrm{eV}$ [see Fig. 1c], a value which compares very well with previous estimates [29-31].

It is worth noting that the full CEF model of Eq. (1), which keeps a description in terms of the magnetic moments and does not perform the projection onto the pseudospin 1/2 states, also predicts the stabilization of the $\psi_{2}$ configuration. The mechanism is however different from order by disorder and the selection results rather from an energetic mechanism: an effective magnetic anisotropy is generated by the molecular field induced admixture between CEF levels. This anisotropy in turn selects the $\psi_{2}$ state and opens a spin gap in $S(Q, \omega)$. This mixing with CEF eigenstates is clearly neglected in model (2) which, by essence, considers the ground doublet only. This study thus suggests that ObD should be considered with care [34].

To conclude, the present study demonstrates that the single crystal time-of-flight measurements allow one to obtain a comprehensive dataset, measuring in "one shot" the whole spin wave dispersion. With the help of a model, quantitative information can be extracted, for instance the determination of the exchange parameters.

\section{3. $\mathbf{T b}_{2} \mathbf{T i}_{2} \mathbf{O}_{7}$}

The pyrochlore lattice has been also widely studied for ferromagnetic interactions combined with Ising spins constrained to lie along the local $\vec{z}_{i}=\langle 111\rangle$ axes $\left(\vec{z}_{i}\right.$ link the center of each tetrahedron to its summits) [35,36]. In that case, the spins write $\vec{S}_{i}=\sigma_{i} \vec{z}_{i}$, which, with $\vec{z}_{i} \cdot \vec{z}_{j}=$ $-\frac{1}{3},(i \neq j)$, leads to the following Hamiltonian:

$$
\mathcal{H}=\sum_{\langle i, j\rangle} \frac{-\mathrm{J}_{z z}}{3} \sigma_{i} \sigma_{j}=\frac{-\mathrm{J}_{z z}}{3} \sum_{\Delta}\left(\sum_{i_{\Delta}} \sigma_{i}\right)^{2}
$$

where $\Delta$ denotes a tetrahedron of the structure and $i_{\Delta}$ runs over the summits of this tetrahedron. Since $J_{z z} \leq$ 0 , the minimization of the energy is ensured by the so called "ice-rule" condition $\sum_{i_{\Delta}} \sigma_{i}=0$, stating that each tetrahedron of the pyrochlore lattice must have, in its ground-state, two spins pointing in and two spins pointing out, in close analogy with the positions of protons of water molecules in real ice [35-37]. There are six possible spin configurations that satisfy the ice rule on a tetrahedron yielding the same ground state degeneracy and the same entropy as in real ice [38] in the thermodynamic limit. One of the main consequences of the ice-rule is the existence of sharp and anisotropic features called "pinchpoints" that are clearly observable in diffuse neutron scattering. The spin ice case is realized in $\mathrm{Ho}_{2} \mathrm{Ti}_{2} \mathrm{O}_{7}$ and $\mathrm{Dy}_{2} \mathrm{Ti}_{2} \mathrm{O}_{7}$ pyrochlores where the effective ferromagnetic interaction results from the dominant dipolar interaction which overcomes the antiferromagnetic nearest neighbor exchange $[39,40]$.

We shall now focus on other remarkable situations produced by "relaxing" the Ising nature of the spins. In this case, appreciable fluctuations between degenerate configurations are restored, resulting in a spin liquid state, a "quantum variant" of spin ices. Current theoretical descriptions are based on the generic pseudospin-1/2 Hamiltonian given by Eq. (2). The states of the pseudospin span the CEF ground doublet $| \pm\rangle$, the Ising exchange constant $J_{z z}$ is responsible for the spin-ice behavior, while the "quantum" transverse terms $J_{ \pm}, J_{z \pm}$ and $J_{ \pm \pm}$introduce fluctuations [9,41-45].

A potential candidate for this particular regime is $\mathrm{Tb}_{2} \mathrm{Ti}_{2} \mathrm{O}_{7}$. It is characterized by an Ising-like anisotropy of the non-Kramers $\mathrm{Tb}^{3+}$ ions along the local $\langle 111\rangle$ axes $[46,47]$. In spite of effective antiferromagnetic interactions leading to a Curie-Weiss temperature of $-13 \mathrm{~K}$ [48], which should drive the system into long-range order $[32,49]$, prior works pointed out a disordered fluctuating ground state down to $20 \mathrm{mK}[50,51]$. Various subsequent studies have suggested complex spin dynamics, where different time and temperature scales coexist, as revealed by muons [52-54], magnetization [55,56] and neutron scattering experiments [57-66]. Recently, power law spin correlations have also been reported [67], bearing some resemblance with the pinch point pattern observed in the aforementioned spin ices. The peculiar form of these elastic correlations has been confirmed by recent triple axis measurements carried out at $4 \mathrm{~F}$ (LLB), shown in Fig. 2a [68].

The observation of low energy fluctuations (in the $0-0.5 \mathrm{meV}$ range), likely corresponding to fluctuations within the pseudospin-1/2 states $| \pm\rangle$, is a central issue. Recently, polarized inelastic neutron scattering experiments have been performed at IN14, that eventually demonstrate that these low energy fluctuations correspond to the superposition of a quasi-elastic response and of 

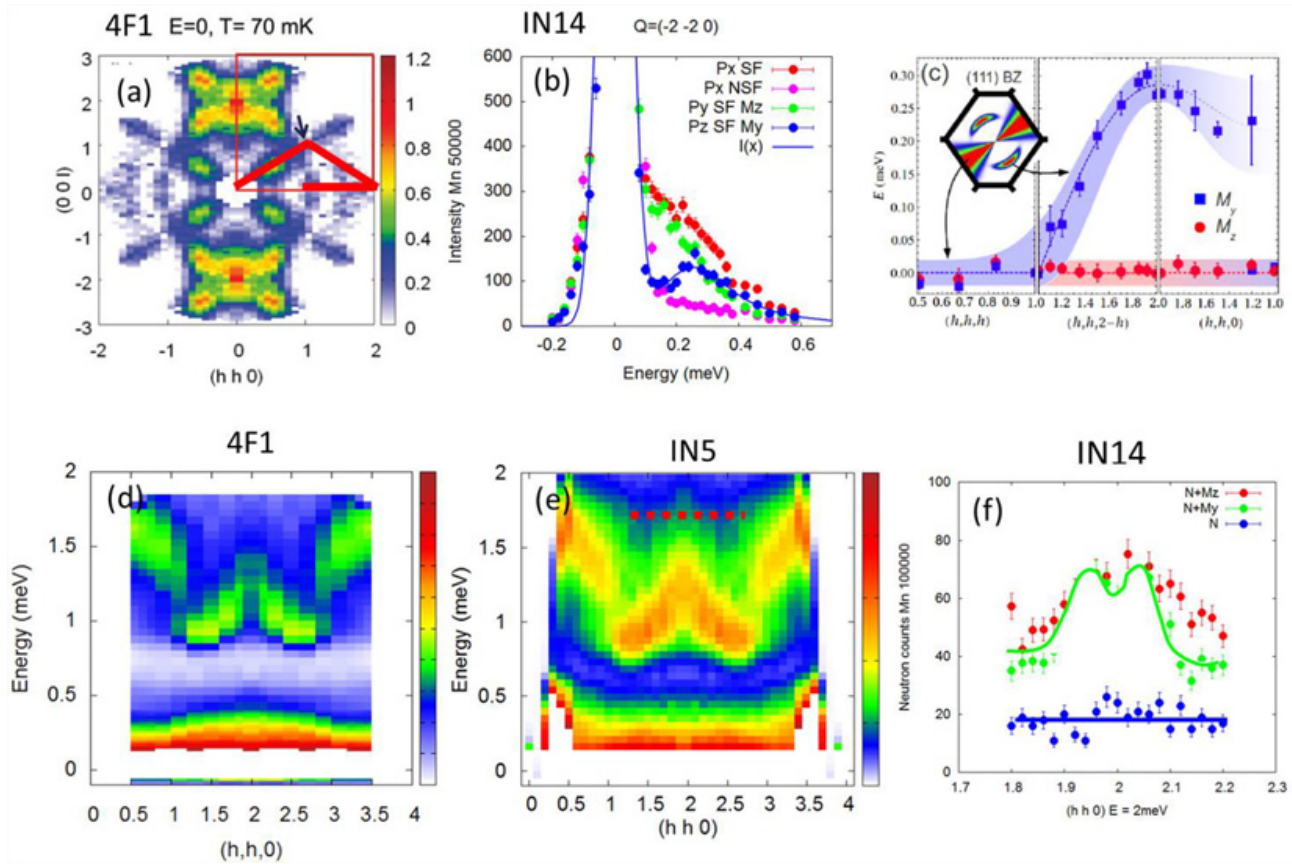

Figure 2. a) Elastic scattering map taken in $\mathrm{Tb}_{2} \mathrm{Ti}_{2} \mathrm{O}_{7}$ at $70 \mathrm{mK}$. Symmetrization has been performed for clarity of the figure. Pinch points are observed at $\mathrm{Q}=(111)$ for instance, bearing resemblance with the situation in classical spin ices. b) IN14 polarized raw data taken at $50 \mathrm{mK}$ in the spin flip channel for different polarizations (Px in red, Py in green and $\mathrm{Pz}$ in blue). The propagating character is detected in the Pz channel. c) Dispersion of the propagating excitation in $\mathrm{Tb}_{2} \mathrm{Ti}_{2} \mathrm{O}_{7}$ from Ref. [68]. The studied Q directions are those shown by red lines on a). d) and e) are respectively 4F (triple axis at LLB) and IN5 (TOF at ILL) raw data showing the modulation of the first excited CEF level around $1.5 \mathrm{meV}$ at base temperature. f) Constant energy scans along the dotted line in e) for different non spin flip polarizations. The scan is expected to go across the acoustic phonon dispersion. Clearly, as the Px channel shows background only, this data is an evidence that the phonon carries some magnetic intensity [78].

a propagating excitation [68]. Figures $2 \mathrm{~b}$ and $\mathrm{c}$ show representative scans as well as the dispersion of this excitation within the Brillouin zone. The contribution of polarized neutrons was very important to disentangle the propagating excitation, observable in the spin-flip $P \| z$ channel [blue points in the scan of Fig. 2b] from the additional quasi-elastic signal seen in the spin flip $P \perp Q$ channel (green points).

It should be stressed that the existence of this low energy signal leads to very strong conclusions about the physics of $\mathrm{Tb}_{2} \mathrm{Ti}_{2} \mathrm{O}_{7}$ : because of the intrinsic properties of non-Kramers magnetic doublets (like the $\mathrm{Tb}^{3+}$ ground state in $\left.\mathrm{Tb}_{2} \mathrm{Ti}_{2} \mathrm{O}_{7}\right)$, the matrix element $\langle+|\vec{J}|-\rangle$ is zero [69]. As a result, since the neutron cross section is proportional to $|\langle+|\vec{J}|-\rangle|^{2}$, no neutron signal is expected. Moreover, low energy fluctuations cannot originate from the exchange/dipole interaction. The experimental observations thus call for further interpretation. Assuming that a fluctuating molecular field mixes the ground and excited CEF states, non-zero matrix elements might in principle be restored $[46,70]$. However, as long as the exchange terms are one order of magnitude weaker than the gap $\Delta$ to the first CEF excited states, the perturbed wave function should not depart significantly from $| \pm\rangle[71,72]$. This is the reason why research now keeps looking towards new physical ingredients that may directly couple the $| \pm\rangle$ states. Recent studies argue that a coupling between quadrupolar moments $\mathcal{Q}$ may play a significant role, since $\langle+|\mathcal{Q}|-\rangle$ is non zero. As a result, the degeneracy of the $| \pm\rangle$ states should be lifted, providing a first explanation for the low energy dispersing signal. A model based on the most simple on-site quadrupolar term has been proposed, phenomenologically connected with a possible tetragonal distortion precursor to a $T \simeq 0$ Jahn-Teller transition [53,61,70-72,75-77]. Despite being rather successful in explaining a number of experimental results [61,71-74], this model is however incomplete as it predicts a CEF singlet state on each site, a feature which is not compatible with the existence of elastic correlations.

At slightly larger energies, $\hbar \omega \sim 1-2 \mathrm{meV}$, the inelastic response is dominated by the first $\mathrm{CEF}$ excitation located at $\Delta \approx 1.5 \mathrm{meV}$ [see Figs. $2 \mathrm{~d}$ and e]. The line shape of this CEF excitation is much more complicated than a single dispersionless mode and very likely contains several excitation modes. The line shape is modulated already at $10 \mathrm{~K}$ and down to the base temperature of $50 \mathrm{mK}$, because of the interactions between $\mathrm{Tb}^{3+}$ magnetic moments [32]. In a very narrow range of scattering vectors $\mathbf{Q}$ close to crystalline zone centers, such as $(1,1,1)$ and $(2,2,0)$, an unexpected upturn of the dispersion is observed [see Figs. 2d and e]. This upturn arises in the region of reciprocal space where a crossing occurs between the crystal field line and the acoustic phonon branch stemming from the zone centers. Very recently, Tom Fennell et al have shown that this phonon is best described by a magneto-elastic mode [78]. The authors argue that in the spin liquid state, it is coupled to the excited crystal field level forming a hybrid excitation 
and suggesting that $\mathrm{Tb}_{2} \mathrm{Ti}_{2} \mathrm{O}_{7}$ is a "magnetoelastic spin liquid" (yet the hybridization mechanism remains to be further elucidated). To illustrate this point, Fig. $2 f$ shows a cut along $\langle h, h, 0\rangle$, across the dispersion of the phonon mode, taken at an energy transfer of $1.8 \mathrm{meV}$. This experiment was carried out with polarized neutron analysis, in collaboration with the authors of Ref. [78]. In the $P \| Q$ configuration, the non-spin-flip signal shows no intensity while it actually does in the $P \perp$ $Q$ and $P \| z$ configurations. Clearly, the conclusion is that the phonon carries some magnetic intensity. The magneto-elastic coupling responsible for the phonon and CEF anomalies could be the driving force leading to the wanted effective interactions between quadrupoles. Indeed, this magneto-elastic coupling would involve in the Hamiltonian a term of the form $u . \mathcal{Q}$, where $u$ denotes the phonon displacement operator. Integrating out the phonon variables could then result in an effective interaction between quadrupoles mediated by phonons [79-81]. Note that there are additional clues in favor of a strong dynamical spin-lattice coupling: structural fluctuations below $15 \mathrm{~K}$ observed by high resolution $\mathrm{X}$ diffraction [82], giant magneto-striction [83] and the instability of the spin liquid state versus pressure and stress [84], all of which have been reported recently, but no static distortion has been observed so far [85].

\section{Conclusion}

To conclude, single crystal time-of-flight measurements now yield very detailed data, which can be confronted to spin dynamics simulations. Measurements in variable conditions of pressure, magnetic field, temperature, and especially polarized techniques continue however to be the preserve of triple axis spectrometers. In this respect both techniques are fully complementary.

In the context of pyrochlores, the study of the spin dynamics in $\mathrm{Er}_{2} \mathrm{Ti}_{2} \mathrm{O}_{7}$ demonstrates that quantitative information can be extracted from single crystal TOF data, provided models are available. This supposes a close connection with theoreticians. In $\mathrm{Tb}_{2} \mathrm{Ti}_{2} \mathrm{O}_{7}$, the combination of $\mathrm{TOF}$ and polarized triple axis measurements demonstrate the existence of a low energy propagating excitation emanating from the spin liquid ground state. Anomalies of the phonon modes, as well as of the first CEF level, suggest a strong dynamical coupling to the lattice.

We acknowledge E. Lhotel, L. Jaubert, P. McClarty, Michel Gingras and Jeff Rau for fruitful discussions. We also thank S. Turc and P. Boutrouille (cryogeny group at ILL and LLB respectively) for their technical help while setting up the magnet and the dilution fridge.

\section{References}

[1] Introduction to Frustrated Magnetism, edited by C. Lacroix, P. Mendels, and F. Mila (Springer-Verlag, Berlin, 2011)

[2] M. J. P. Gingras and P. A. McClarty Rep. Prog. Phys. 77, 056501 (2014)
[3] S. T. Bramwell and M. J. P. Gingras, Science 294, 1495 (2000)

[4] J. S. Gardner, M. J. P. Gingras and J. E. Greedan, Rev. Mod. Phys. 82, (2010) 53

[5] S. T. Bramwell, M. J. P. Gingras, and J. N. Reimers, J. Appl. Phys. 75, 5523 (1994)

[6] J. M. D. Champion and P. C. W. Holdsworth, J. Phys.: Condens. Matter 16, S665 (2004)

[7] P. A. McClarty, P. Stasiak, and M. J. P. Gingras, Phys. Rev. B 89, 024425 (2014)

[8] M. E. Zhitomirsky, M. V. Gvozdikova, P. C. W. Holdsworth, and R. Moessner, Phys. Rev. Lett. 109, 077204 (2012)

[9] L. Savary, K. A. Ross, B. D. Gaulin, J. P. C. Ruff, and L. Balents, Phys. Rev. Lett. 109, 167201 (2012)

[10] A. W. C. Wong, Z. Hao, and M. J. P. Gingras, Phys. Rev. B 88, 144402 (2013)

[11] J. Oitmaa, R. R. P. Singh, B. Javanparast, A. G. R. Day, B. V. Bagheri, and M. J. P. Gingras, Phys. Rev. B 88, 220404 (2013)

[12] H. Yan, O. Benton, L. D. C. Jaubert, and N. Shannon, F arXiv:1311.3501 (2013)

[13] M. E. Zhitomirsky, P. C. W. Holdsworth, and R. Moessner Phys. Rev. B 89, 140403(R) (2014)

[14] J. D. M. Champion, M. J. Harris, P. C. W. Holdsworth, A. S. Wills, G. Balakrishnan, S. T. Bramwell, E. Cizmar, T. Fennell, J. S. Gardner, J. Lago, D. F. McMorrow, M. Orendac, A. Orendacova, D. McK. Paul, R. I. Smith, M. T. F. Telling, and A. Wildes, Phys. Rev. B. 68, 020401(R), (2003)

[15] J. Villain, R. Bidaux, J.-P. Carton, and R. Conte, J. Phys. 41, 1263 (1980)

[16] E. F. Shender, Sov. Phys. JETP 56, 178 (1982)

[17] T. Yildirim, Turk. J. Phys. 23, 47 (1999)

[18] W. J. Blöte, R.F. Wielinga and W. J. Huiskamp, Physica 43, 549 (1969)

[19] M. J. Harris, S. T. Bramwell, T. Zeiske, D. F. McMorrow, and P. J. C. King, J. Magn. Magn. Mater. 177, 757 (1998)

[20] R. Siddharthan, B. S. Shastry, A. P. Ramirez, A. Hayashi, R. J. Cava, and S. Rosenkranz, Phys. Rev. Lett. 83, 1854 (1999)

[21] A. Poole, A. S. Wills, and E. Lelièvre-Berna, J. Phys.: Condens. Matter 19, 452201 (2007)

[22] P. A. McClarty, S. H. Curnoe, and M. J. P. Gingras, Journal of Physics: Conference Series 145, 012032 (2009)

[23] S. Guitteny, S. Petit, E. Lhotel, J. Robert, P. Bonville, A. Forget, and I. Mirebeau, Phys. Rev. B 88, 134408 (2013)

[24] B. G. Wybourne, Spectroscopic Properties of Rare Earths (Interscience, New York, 1965)

[25] The crystal field is modeled by the following coefficients: $B_{20}=616 \mathrm{~K}, B_{40}=2850 \mathrm{~K}, B_{43}=795$ $\mathrm{K}, B_{60}=858 \mathrm{~K}, B_{43}=-494 \mathrm{~K}, B_{66}=980 \mathrm{~K}$, in Weybourne conventions

[26] J. Jensen and A. R. Mackintosh, Rare Earth Magnetism, Clarendon Press, Oxford, 1991

[27] B. Z. Malkin, T. T. A. Lummen, P. H. M. van Loosdrecht, G. Dhalenne, and A.R. Zakirov, J. Phys.: Condens. Matter 22, 276003 (2010) 
[28] J. P. C. Ruff, J. P. Clancy, A. Bourque, M. A. White, M. Ramazanoglu, J. S. Gardner, Y. Qiu, J. R. D. Copley, M. B. Johnson, H. A. Dabkowska, and B. D. Gaulin, Phys. Rev. Lett. 101, 147205 (2008)

[29] S. S. Sosin, L. A. Prozorova, M. R. Lees, G. Balakrishnan, and O. A. Petrenko, Phys. Rev. B 82, 094428 (2010)

[30] P. Dalmas de Réotier, A. Yaouanc, Y. Chapuis, S. H. Curnoe, B. Grenier, E. Ressouche, C. Marin, J. Lago, C. Baines, and S. R. Giblin, Phys. Rev. B 86, 104424 (2012)

[31] K. A. Ross, Y. Qiu, J. R. D. Copley, H. A. Dabkowska, and B. D. Gaulin, Phys. Rev. Lett. 112 057201 (2014)

[32] Y. J. Kao, M. Enjalran, A. Del Maestro, H. R. Molavian, and M. J. P. Gingras, Phys. Rev. B 68, 172407 (2003)

[33] S. Petit, in Collection SFN 12 (2011), published by EDP Sciences, http://dx.doi.org/10. $1051 /$ sfn/201112006

[34] S. Petit et al, to appear in Phys. Rev. B Rapid Comm (2014)

[35] S. T. Bramwell, M. J. Harris, B. C. den Hertog, M. J. P. Gingras, J. S. Gardner, D. F. McMorrow, A. R. Wildes, A. L. Cornelius, J. D. M. Champion, R. G. Melko, and T. Fennell, Phys. Rev. Lett. 87, 047205 (2001); S. T. Bramwell and M. J. P. Gingras, Science 294, 1495 (2001)

[36] C. Castelnovo, R. Moessner, and S. L. Sondhi, Phys. Rev. Lett. 104, 107201 (2010)

[37] H. J. Harris, S. T. Bramwell, D. F. McMorrow, T. Zeiske, K. W. Godfrey Phys. Rev. Lett. 79, 2554 (1997)

[38] A. P. Ramirez et al., Nature 399, (1999) 333

[39] C.L. Henley, Phys Rev. B 71, 014424 (2005)

[40] T. Fennell, P. P. Deen, A. R. Wildes, K. Schmalzl, D. Prabhakaran, A. T. Boothroyd, R. J. Aldus, D. F. McMorrow, S. T. Bramwell, Science 326, 415 (2009)

[41] M. Hermele, M. P. A. Fisher and L. Balents, Phys. Rev. B 69, 064404 (2004)

[42] S. Onoda and Y. Tanaka, Phys. Rev. Lett. 105, 047201 (2010); S. Onoda and Y. Tanaka, Phys. Rev. B 83, 094411 (2011)

[43] L. Savary and L. Balents, Phys. Rev. Lett. 108, 037202 (2012)

[44] O. Benton, O. Sikora and N. Shannon, Phys. Rev. B 86, 075154 (2012)

[45] S.B. Lee, S. Onoda, and Leon Balents, Phys. Rev. B 86, 104412 (2012)

[46] H. R. Molavian, M. J. P. Gingras, and B. Canals, Phys. Rev. Lett. 98, 157204 (2007)

[47] H.B. Cao, I. Mirebeau, A. Gukasov, P. Bonville, and C. Decorse, Phys. Rev. B 82, 104431 (2010)

[48] M. J. P. Gingras, B. C. den Hertog, M. Faucher, J. S. Gardner, S. R. Dunsiger, L. J. Chang, B. D. Gaulin, N. P. Raju, and J. E. Greedan, Phys. Rev. B 62, 6496 (2000)

[49] B. C. den Hertog and M. J. P. Gingras, Phys. Rev. Lett. 84, 3430 (2000)

[50] J. S. Gardner, S. R. Dunsiger, B. D. Gaulin, M. J. P. Gingras, J. E. Greedan, R. F. Kiefl, M. D. Lumsden, W. A. MacFarlane, N. P. Raju, J. E. Sonier,
I. Swainson and Z. Tun, Phys. Rev. Lett. 82, 1012 (1999)

[51] J. S. Gardner, B. D. Gaulin, A. J. Berlinsky, P. Waldron, S. R. Dunsiger, N. P. Raju and J. E. Greedan, Phys. Rev. B 64, 224416 (2001)

[52] J. S. Gardner, A. Keren, G. Ehlers, C. Stock, Eva Segal, J. M. Roper, B. Fak, M. B. Stone, P. R. Hammar, D. H. Reich, and B. D. Gaulin., Phys. Rev. B 68, 180401(R) (2003)

[53] Y. Chapuis, A. Yaouanc, P. Dalmas de Réotier, C. Marin, S. Vanishri, S. H. Curnoe, C. Vaju, and A. Forget, Phys. Rev. B 82, 100402(R) (2010)

[54] A. Yaouanc, P. Dalmas de Réotier, Y. Chapuis, C. Marin, S. Vanishri, D. Aoki, B. Fak, L.-P. Regnault, C. Buisson, A. Amato, C. Baines, and A. D. Hillier, Phys. Rev. B 84, 184403 (2011)

[55] E. Lhotel, C. Paulsen, P.D. deRéotier, A. Yaouanc, C. Marin and S. Vanishri, Phys. Rev. B 86 020410(R) (2012)

[56] S. Legl, C. Krey, S. R. Dunsiger, H.A. Dabkowska, J.A. Rodriguez, G. M. Luke, and C. Pfleiderer, Phys. Rev. Lett. 109, 047201 (2012)

[57] Y. Yasui, M. Kanada, M. Ito, H. Harashina, M. Sato, H. Okumura, K. Kakurai, and H. Kadowaki, J. Phys. Soc. Jpn. 71, 599 (2002)

[58] I. Mirebeau, P. Bonville, M. Hennion, Phys. Rev. B 76, 184436 (2007)

[59] K. C. Rule, G. Ehlers, J. R. Stewart, A. L. Cornelius, P. P. Deen, Y. Qiu, C. R. Wiebe, J. A. Janik, H. D. Zhou, D. Antonio, B. W. Woytko, J. P. Ruff, H. A. Dabkowska, B. D. Gaulin and J. S. Gardner, Phys. Rev. B 76, 212405 (2007)

[60] K. C. Rule, G. Ehlers, J. S. Gardner, Y. Qiu, E. Moskvin, K. Kiefer and S. Gerischer, J. Phys.: Condens. Matter 21, 486005 (2009)

[61] S. Petit, P. Bonville, I. Mirebeau, H. Mutka, and J. Robert, Phys. Rev. B 85, 054428 (2012)

[62] T. Taniguchi, H. Kadowaki, H. Takatsu, B. Fak, J. Ollivier, T. Yamazaki, T. J. Sato, H. Yoshizawa, Y. Shimura, T. Sakakibara, T. Hong, K. Goto, L. R. Yaraskavitch, and J. B. Kycia, Phys. Rev. B 87, 060408R (2010)

[63] B. D. Gaulin, J. S. Gardner, P. A. McClarty and M. J. P. Gingras, Phys. Rev. B. 84, 140402 (2011)

[64] H. Takatsu, H. Kadowaki, Taku J. Sato, J. W. Lynn, Y. Tabata, T. Yamazaki, and K. Matsuhira, J. Phys.: Condens. Matter 24, 052201 (2012)

[65] L. Yin, J. S. Xia, Y. Takano, N. S. Sullivan, Q. J. Li, and X. F. Sun Phys. Rev. Lett. 110, 137201 (2013)

[66] K. Fritsch, K. A. Ross, Y. Qiu, J. R. D. Copley, T. Guidi, R. I. Bewley, H. A. Dabkowska, and B. D. Gaulin , Phys. Rev. B 87, 094410 (2013)

[67] T. Fennell, M. Kenzelmann, B. Roessli, M. K. Haas, and R. J. Cava, Phys. Rev. Lett. 109, 017201 (2012)

[68] S. Guitteny, J. Robert, P. Bonville, J. Ollivier, C. Decorse, P. Steffens, M. Boehm, H. Mutka, I. Mirebeau, and S. Petit Phys. Rev. Lett. 111, 087201 (2013)

[69] K. A. Mueller, Phys. Rev. 171350 (1967)

[70] S. H. Curnoe, Phys. Rev. B 78, 094418 (2008) 
[71] P. Bonville, I. Mirebeau, A. Gukasov, S. Petit, J. Robert, Phys. Rev. B 84, 184409 (2011)

[72] S. Petit, P. Bonville, I. Mirebeau, H. Mutka, and J. Robert, Phys. Rev. B 85, 054428 (2012)

[73] P. Bonville et al. Phys. Rev. B 89, 085115 (2014)

[74] A Sazonov et al, Phys. Rev. B 88, 184428 (2013)

[75] L. G. Mamsurova, K. S. Pigal'skii, K. K. Pukhov, JETP Lett. 43, 755 (1986)

[76] K. C. Rule, P. Bonville, J. Phys. Conference Series 145, 012027 (2009)

[77] P. Bonville, I. Mirebeau, A. Gukasov, S. Petit and J. Robert, J. Phys.: Conf. Series 32, 012006 (2011)

[78] T. Fennell, M. Kenzelmann, B. Roessli, H. Mutka, J. Ollivier, M. Ruminy, U. Stuhr, O. Zaharko, L. Bovo, A. Cervellino, M.K. Haas, and R.J. Cava, Phys. Rev. Lett. 112, 017203 (2014)

[79] G. A. Gehring and K. A. Gehring, Reports on progress in physics 38, 1 (1975)
[80] R.J. Birgeneau, J.K. Kjems, G. Shirane, L.G. Van Uitert, Phys. Rev. B 10, 2512 (1974)

[81] P. Thalmeier and P. Fulde, Phys. Rev. Lett. 49, 1588 (1982)

[82] J. P. C. Ruff, B. D. Gaulin, J. P. Castellan, K. C. Rule, J. P. Clancy, J. Rodriguez and H. A. Dabkowska, Phys. Rev. Lett. 99, 237202 (2007)

[83] J. P. C. Ruff, Z. Islam, J. P. Clancy, K. A. Ross, H. Nojiri, Y. H. Matsuda, H. A. Dabkowska, A. D. Dabkowski, and B. D. Gaulin, Phys. Rev. Lett. 105, 077203 (2010)

[84] I. Mirebeau, I. N. Goncharenko, P. Cadavez-Peres, S. T. Bramwell, M. J. P. Gingras and J. S. Gardner, Nature 420, 54 (2002); I. Mirebeau, I.N. Goncharenko, G. Dhalenne and A. Revcolevschi, Phys. Rev. Lett. 93, 187204 (2004)

[85] K. Goto, H. Takatsu, T. Taniguchi and H. Kadowaki, J. Phys. Soc. Jpn. 81, 015001 (2012) 